\title{
Impacto de PH del fijador, tiempo de fijación, tiempo previo sin fijación en la expresividad de marcadores moleculares de inmunohistoquímica
}

Impact of fixer PH, fixation time, previous time without fixation on the expressivity of molecular markers of immunohistochemistry

1 Milton David Tapia Medina inmunohistoquímica. Anatomía Digital, 5(1), 6-25.

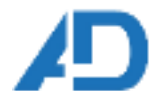

Ciencia Digital

ANATOMÍA DIGITAL, es una Revista Electónica, Trimestral, que se publicará en soporte electrónico tiene como misión contribuir a la formación de profesionales competentes con visión humanística y crítica que sean capaces de exponer sus resultados investigativos y científicos en la misma medida que se promueva mediante su intervención cambios positivos en la sociedad. https://anatomiadigital.org

La revista es editada por la Editorial Ciencia Digital (Editorial de prestigio registrada en la Cámara Ecuatoriana de Libro con No de Afiliación 663) www.celibro.org.ec

Esta revista está protegida bajo una licencia Creative Commons


Palabras claves: fase preanalítica, procesamiento histotecnológico inmunohistoquímica, fijación, fijador, $\mathrm{pH}$.

\section{Keywords:}

preanalytical phase, immunohistochemical histotechnology processing, fixation, fixer, ph.
Resumen

El desarrollo exponencial de la inmunohistoquímica la ha convertido en una pieza fundamental para el diagnóstico, pronóstico y tratamiento de distintas patologías, por esto el conocimiento del procesamiento histotecnológico permitirá la detección oportuna de aquellas variables en las fases del proceso que influyen en la expresión de los inmuno marcadores que generarían errores diagnósticos, pérdida de recursos económicos y biológicos. OBJETIVO: establecer las condiciones de manejo preanalítico: $\mathrm{pH}$ del Fijador, tiempo de fijación, tiempo previo sin fijación, en la expresividad de marcadores de inmunohistoquímica. MÉTODO: se realizó una revisión documental actualizada de publicaciones en la base de datos de revistas indexadas PUBMED y otras con relación al proceso pre analítico, procesamiento de tejidos en el laboratorio de Anatomía Patológica, técnica de inmunohistoquímica, relación existente entre el resultado de marcadores de inmunohistoquímica y las variaciones de $\mathrm{pH}$ de fijador, tiempos de fijación y tiempos previos a fijación, desde el año 2011, obteniéndose 57 publicaciones, las cuales se consolidaron y se presentaron en este estudio. CONCLUSIÓN: se demuestra la importancia del proceso pre analítico en sus diferentes fases, y su efecto para obtención de laminillas adecuadas para un correcto análisis. Tiempos de fijación y fijadores en $\mathrm{pH}$ óptimos son vitales en la fase pre analítica.

\section{Abstract}

The exponential development of immunohistochemistry has become a fundamental piece for the diagnosis, prognosis and treatment of different pathologies, for this reason, the knowledge of histotechnology processing will allow the timely detection of those variables in the phases of the process that influence the expression of immunomarkers that would generate diagnostic errors, loss of economic and biological resources. OBJECTIVE: to establish the preanalytical handling conditions: fixative $\mathrm{pH}$, fixation time, previous time without fixation, in the expression of immunohistochemical markers. METHODS: an updated documentary review of publications in the database of indexed journals PUBMED and others was carried out in relation to the pre-analytical process, tissue processing in the 
Pathological Anatomy laboratory, immunohistochemical technique, relationship between the result of markers of Immunohistochemistry and variations in fixative $\mathrm{pH}$, fixation times and times prior to fixation since 2011, obtaining 57 publications which were consolidated and presented in this study. CONCLUSION: the importance of the pre-analytical process in its different phases is demonstrated, and its effect to obtain adequate lamellae for a correct analysis. Optimum fixation and fixation times at $\mathrm{pH}$ are vital in the pre-analytical phase.

\section{Introducción}

La inmunohistoquímica, es un estudio complementario en la rama de la patología que tiene como objetivo la detección de un antígeno especifico y de su localización en la arquitectura celular, esto se logra a través de una reacción antígeno anticuerpo especifica (Alarcón \& Plaza, 2015; Ter \& Verani, 2017).

Los inicios de la inmunohistoquímica datan de la década de los 40 , no es hasta finales de los 70s cuando existe un crecimiento exponencial de su uso, a la par del desarrollo de marcadores moleculares (Vaquero, 2012; Teruya-Feldstein, 2011; Moorchung \& Consultant, 2019), de igual manera la estandarización de normas internacionales que intentan reducir al mínimo factores que alterarían el correcto resultado (Teruya-Feldstein, 2011). En Estados Unidos los laboratorios están bajo estricta regulación de la FDA quien verifica el desempeño, eficacia y precisión de las pruebas diagnósticas y la implementación de protocolos de procesos (Fitzgibbons et al., 2014; Dagher et al., 2019; Maximilian, 2021). En la actualidad los estudios histopatológicos cuyo diagnóstico sea de un tumor maligno o un tumor benigno con sospecha de posible malignidad bajo el criterio del especialista con pruebas de inmuno histoquímica pueden ser confirmados (Fitzgibbons et al., 2014). Al momento se investigan nuevas sustancias que son producidas por células tumorales y su presencia pueden ser demostradas por pruebas de inmunohistoquímica (Atkins et al., 2004), por lo que esta técnica ha tenido un gran impacto en todas las áreas de la patología, a pesar del auge de otras técnicas como citometría de flujo o técnicas moleculares que han aportado grandes contribuciones al área de la patología quirúrgica no han podido desplazar a la inmunohistoquímica en el uso cotidiano (Miller, 2011; Donovan \& Cordon, 2016).

La aplicación de inmunohistoquímica, es indispensable para el estudio de diferentes tejidos y sus resultados son fundamentales para el diagnóstico, pronóstico y posterior 
tratamiento de los pacientes, por este motivo es importante antes de emitir un diagnóstico (Parra-Medina \& Polo, 2017), demostrar la eficacia y precisión de las pruebas (Hammond et al., 2010). Recientes estudios demuestran que existe variabilidad en la interpretación de resultados entre diferentes laboratorios, producto de fallos en el seguimiento de protocolos preanalíticos y analíticos (Fitzgibbons et al., 2014). Un factor fundamental que induce a resultados erróneos obedecen a fallas en el proceso pre analítico (Fitzgibbons et al., 2014), tales factores son capaces de introducir artefactos o interferir en un resultado exitoso generando de esta manera pérdida de material, recursos económicos y errores diagnósticos (Vaquero, 2012). El diagnóstico anatomo patológico tiene como objetivo la categorización de los tumores , especialmente en metástasis en busca del tumor primario, así como en tumores indiferenciados (Bellizzi, 2020; Shivani \& Lin, 2017). Por lo que las pruebas de inmunohistoquímica son herramientas de gran utilidad en la práctica diaria para la búsqueda del linaje tumoral, objetivo que con solo la morfología sería imposible alcanzar (Bellizzi, 2020). El impacto de las condiciones del manejo pre analítico es determinante, factores como el pH del fijador, el tiempo de fijación y el tiempo previo sin fijación inciden notablemente sobre la expresividad de los marcadores de inmunohistoquímica.

\section{Metodología}

Se realizó una revisión documental actualizada de publicaciones en la base de datos de revistas indexadas PUBMED y otras con relación al proceso pre analítico, procesamiento de tejidos en el laboratorio de Anatomía Patológica , técnica de inmunohistoquímica , relación existente entre el resultado de marcadores de inmunohistoquímica y las variaciones de $\mathrm{pH}$ de fijador, tiempos de fijación y tiempos previos a fijación, desde el año 2011, obteniéndose 57 publicaciones, las cuales se consolidaron y se presentaron en este estudio.

\section{Resultados}

Luego de la búsqueda inicial, se seleccionaron 57 artículos científico que se ajustaban a los criterios de selección, considerando las variables y objetivos del tema de estudio. La figura 1 resume el proceso preanalítico con recomendaciones para una adecuada expresividad de marcadores de inmunohistoquímica en sus distintas fases. 


\section{Figura 1}

Procesamiento preanalítico

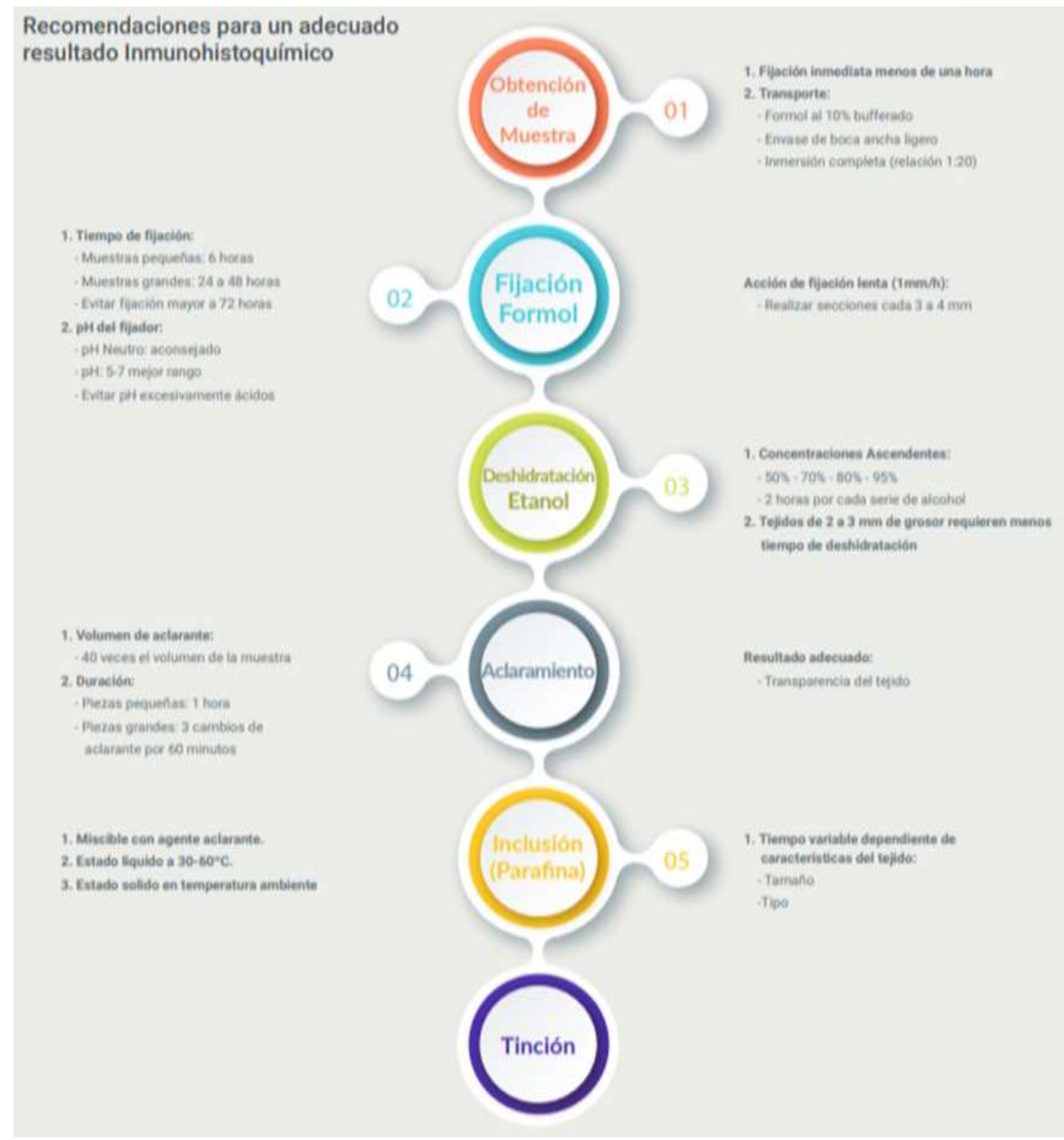

Desarrollo

Concepto de Proceso pre analítico, el desarrollo, perfeccionamiento y estandarización de las técnicas histológicas han supuesto en el mundo de la patología, la piedra angular en el diagnóstico preciso de las distintas entidades nosológicas, que debido a sus características, algunas de ellas tan sutiles ,se convierten en verdaderos retos diagnósticos, que sin la ayuda de una laminilla con un adecuado y minucioso proceso pre analítico sería imposible alcanzar (Alarcón \& Plaza, 2015). 
El preanálisis a pesar de su importancia todavía sigue siendo un enigma para muchos patólogos, lo que genera dificultades para la identificación de los problemas durante el proceso (Chung et al., 2018).

Se entiende como proceso pre analítico, a la secuencia de pasos que inician desde el momento en que el espécimen es extraído hasta el momento que la laminilla es evaluada por el especialista (Lester, 2014). Durante este lapso tenemos etapas muy bien caracterizadas que va desde la extracción del espécimen hasta su procesamiento, este proceso es crucial tanto para una buena evaluación en cortes par hematoxilina eosina, como para pruebas complementarias como la inmuno histoquímica o el estudio molecular (Stradleigh \& Ishida, 2015; Bloom et al., 2019; Biobank, 2018).

Transporte del espécimen: Del centro quirúrgico al laboratorio de patología, el primer punto crucial en el proceso pre analítico es el transporte de los especímenes, tema poco conocido por parte del cirujano, es de vital importancia la socialización de protocolos de estandarización para un adecuado manejo de las muestras (Gaffney et al., 2018).

Todas las salas de operación deben poseer en sus quirófanos, envases adecuados con formol bufferado al 10\%, lo cual va a garantizar la preservación de la muestra y su correcta fijación, dichos envases deben ser de materiales ligeros, de boca grande y que permitan la inmersión completa del espécimen, caso contrario el proceso de fijación será heterogéneo (Lester, 2014).

El inicio del proceso de autolisis y degeneración de las proteínas tanto como ADN y ARN comienza inmediatamente tras la extracción de la muestra, lo cual se conoce como tiempo de isquemia, para fines didácticos se la puede clasificar en isquemia caliente, definida como el tiempo en el que el órgano extraído mantiene la temperatura corporal después de la supresión del flujo sanguíneo, dicha fase en varias investigaciones no compromete el estudio posterior (Bass et al, 2014; Bonin \& Stanta, 2020; Neumeister \& Juhl, 2018). Una segunda fase denominada isquemia fría catalogada como el tiempo entre la extracción de la pieza quirúrgica y su fijación representa un obstáculo para el diagnóstico, tanto para el análisis morfológico como para la aplicación de pruebas complementarias como inmunohistoquímica y biología molecular ( Bass et al, 2014; Mathieson et al., 2019) motivo por el cual deben ser sumergidos lo más pronto posible en formol al $10 \%$ bufferado para detener este proceso.

Lamentablemente no existe un protocolo universal de manejo pre analítico de muestras, algunas investigaciones recomiendan, la implementación de instalaciones de laboratorios de patología dentro de los cetros quirúrgicos, lo cual facilitaría el transporte, su valoración macroscópica en fresco y si es necesario toma de tejidos para estudios moleculares (Comanescu et al., 2012; Socias et al., 2019). Uno de los criterios que si se encuentran estandarizados es la sección del espécimen para una correcta penetración del fijador, 
cortes cada 3 a $4 \mathrm{~mm}$ en muestras mayores de $2 \mathrm{~cm}$, son adecuados para este propósito. (Lester, 2014; Comanescu et al., 2012).

Procesamiento histotecnológico, se denomina procesamiento histotecnológico al conjunto de procesos establecidos que permiten la correcta valoración de los tejidos, manteniendo las facultades naturales del espécimen estudiado (Lester, 2014), este proceso no es rígido y más bien se amolda y modifica de acuerdo a las necesidades de los laboratorio de anatomía patológica (Megías \& Molist, 2016). En líneas generales este proceso se encuentra compuesto por las siguientes etapas: Fijación, Deshidratación, Aclaramiento, Inclusión y Tinción. Cada una de ellas tiene un papel fundamental ya que un procesamiento defectuoso en cualquier punto incidirá de manera dramática en el producto final (Dey, 2018) El presente artículo de revisión tiene como objetivo analizar detalladamente las distintas etapas del procesamiento con énfasis en la fijación de los tejidos.

Fijación, el componente principal de las estructuras de los seres vivos vertebrados son las proteínas y ácidos nucleicos que componen la materia prima de las macro moléculas y tejidos, estas proteínas están conformados por cantidades variables de carbohidratos, que gracias a su conformación molecular hidrofílica captan el agua (Lester, 2014).

Existen distintos fijadores para especímenes biológicos, clasificados por las siguientes características: naturaleza de la fijación, sus propiedades químicas, componentes que los conforman y su acción sobre las pretinas del tejido (Dey, 2018; Anvari \& Gharib, 2021).

El principal objetivo de las sustancias de fijación es evitar la disolución por el agua y otros líquidos, provocando resistencia a los tejidos, para impedir su descomposición, gracias a la inactivación de enzimas lisosomales y la estabilización de las estructuras dentro de las células y entre ellas (Lester, 2014), por lo tanto el proceso de fijación permite mantener las características del tejido en estudio lo más cercanas a su estado de un órgano funcionante (Dey, 2018).

Por definición las soluciones de fijación producen un cambio físico y químico de las estructuras generando alteración de la permeabilidad de la membrana celular a macromoléculas, como la parafina.

En el mercado existe una amplia variedad de fijadores, unos más populares que otros, cada uno de ellos con ventajas y desventajas. El fijador ideal para varias guías y estudios debe ser barato, de fácil uso, que no represente un peligro para la salud del operario, sea rápido y sobre todo que preserve las características de los tejidos (Dey, 2018).

El proceso de fijación va a generar ciertos cambios específicos en el tejido los cuales son de vital importancia conocer. Uno de ellos es la alteración del volumen. Algunos fijadores producen edema, otros como el formol o el glutaraldehido generan un estado de 
contracción de volumen del 33\% del tejido (Dey, 2018). Bahr y colaboradores publicaron en 1955 un estudio en el cual demostraron que la contracción del volumen es inversamente proporcional al grado de concentración de formol (Dey, 2018). Otros fijadores interfieren con la tinción por ejemplo el tetroxido de osmium que inhibe la captación de hematoxilina y eosina (Lester, 2014; Dey, 2018). Incluso se han reportado cambios morfológicos en la valoración óptica de los tejidos sobre todo en la densidad de los núcleos celulares (Dey, 2018).

Existen ciertos lineamientos establecidos para obtener una fijación adecuada. Por ejemplo el volumen necesario de fijador indispensable para lograr una preservación óptima del tejido que debe superar de 15 a 20 veces el volumen del tejido (Lester, 2014). Conocer la velocidad de penetración del fijador es de gran utilidad, esta tiende a ser lenta, $0,1 \mathrm{~cm}$ por hora, por lo que el espécimen debe ser seccionado en cortes de 3 a $5 \mathrm{~mm}$ de grosor. para lograr una fijación uniforme (Dey, 2018).

Tipos de fijadores, existe una amplia variedad de soluciones fijadoras disponibles en el mercado, algunas de ellas con actividad coagulante por ejemplo el sulfato de zinc que facilita la exposición de sitios antigénicos por la formación de macro poros, otros sin actividad coagulantes como el formaldehido que tiene la capacidad de modificar los sitios antigénicos por lo que puede suprimir la inmunotinción, se han creado otras sustancias de fijación con características coagulantes y no coagulantes (Cards, 2018). La elección del fijador debe ser cautelosa porque puede influir en la aplicación posterior de exámenes complementarios.

El uso de formaldehido bufferado al $10 \%$ es recomendada para estudios rutinarios y valoraciones por inmunohistoquímica (Rourke \& Padula, 2016). El uso de fijadores como glutaraldehido o tetroxido de osmium se recomiendan para tejidos que serán valorados en microscopía electrónica (Dey, 2018).

Por ser el formaldehido el fijador más utilizado en los laboratorios de patología la revisión bibliográfica expondrá sus características más importantes.

Formaldehido, su uso se ha generalizado y estandarizado a nivel mundial para el manejo de las muestras, se comercializa como formaldehido al $40 \%$ contiene también metanol al 10\% cuya función es la de retardar la formación de polímeros pesados (Lester, 2014; Donczo \& Guttman, 2018).

El formaldehido actúa en distintos puntos de las cadenas proteicas, generando grupos hidroximetil, que desencadena una reacción de enlaces cruzados con formación posterior de puentes de metileno transformando al tejido en insoluble, esta reacción es estable y prolongada (Dey, 2018; Thway et al., 2017). 
Ha sido usada por más de 50 años, normalmente en concentraciones al 10\% bufferado, para mantener un $\mathrm{pH}$ neutro y una presión osmótica similar al medio externo de las células, evitando una contracción y endurecimiento excesivos (Lester, 2014; Dey, 2018). Es el fijador de elección en la mayoría de establecimientos médicos para el procesamiento, es económico y la preparación es sencilla (Dey, 2018; Schmeller et al., 2019).

Las desventajas de este fijador son entre otras su actividad lenta de penetración, de 12 a $24 \mathrm{~h}$ para piezas pequeñas, la incapacidad de preservar los mucopolisacaridos ácidos, la generación de artefactos morfológicamente visibles en tejidos altamente vascularizados. ser muy irritante de mucosas y potencialmente carcinogénico (Dey, 2018; Mastracci et al., 2019; Metovic et al., 2018).

Para poder preservar la ultraestructura de la muestra, en varios estudios de investigación recomiendan mantener un rango de $\mathrm{pH}$ entre 7.2 - 7.4 para evitar el daño celular (Uguen \& Guibourg, 2016).

No hay lineamientos claros en relación con el tiempo, se recomienda individualizar dependiendo del tejido. Se considera que una óptima fijación requiere un minino de 6 a 8 horas y un máximo de 72 horas, se debe tener especial cuidado con la sobre fijación (Ammerlaan et al., 2018).

Deshidratación, luego de la fijación del tejido se procede a la deshidratación, cuyo objetivo fundamental es la eliminación del agua proveniente del espécimen, este proceso se realiza mediante el uso de alcoholes u otras sustancias capaces de preservar la estructura celular y no interferir con los pasos siguientes del procesamiento de los tejidos (Dey, 2018; Montuenga \& Calvo, 2014).

Comúnmente se utiliza Alcohol etílico en concentraciones ascendentes (50.70,80,95 \%), para evitar el daño, debido a que cambios bruscos en la deshidratación ocasionan retracción (Lester, 2014). No existen protocolos estrictos en el número y porcentajes, de alcoholes a utilizar, varias guías dejan estos parámetros al criterio del tecnólogo (Montuenga \& Calvo, 2014).

El tiempo que deben permanecer los tejidos en los alcoholes es fundamental, va a depender del volumen de fragmentos que están en proceso y de su contenido de agua. Debemos recordar que muestras con grosores de entre 2 a $3 \mathrm{~mm}$ requieren menos tiempo de deshidratación en comparación con tejidos con grosor mayor a $5 \mathrm{~mm}$ (Dey, 2018). La exposición excesiva puede ocasionar un endurecimiento de los tejidos (Atkins et al., 2004), Sería deseable que en el proceso se individualicen los especímenes de acuerdo con su volumen y características para evitar los efectos indeseables que ocasiona una exposición prolongada a los alcoholes (Dey, 2018). 
Agentes deshidrantes, el etanol es el agente deshidrante más utilizado en los laboratorios de patología por ser un agente de rápido y eficaz efecto. Se recomienda la individualización de las concentraciones ascendentes del deshidratante, ya que tejidos delicados pueden necesitar concentraciones iniciales de 30\% (Dey, 2018).

Existen otros tipos de agentes deshidratantes en el mercado tales como el Metanol, Dioxane, Etilen Glycol o el Alcohol isopropil que pueden ser utilizados pero debido a sus desventajas comparadas con el Etanol, como el alto costo , rápida evaporación o alto riesgo para el personal de laboratorio han pasado a segundo plano (Dey, 2018).

Aclaramient, el objetivo de este proceso es la sustitución de la sustancia deshidratante por una sustancia miscible en el cual se pueda disolver el medio de inclusión (Montuenga \& Calvo, 2014). Todos los agentes aclarantes tienen un índice de refracción que le permite dar al tejido, desprovisto de agua una apariencia clara, la apariencia opaca en la visualización del tejido se traduce como un proceso de deshidratación incompleto (Dey, 2018).

Existen ciertas variables que pueden alterar el efecto adecuado del aclarante, como son tejidos grandes, tipo de procesador que se utiliza, tiempos de deshidratación inadecuados o temperatura subóptima entre otros (Dey, 2018).

Uno de los aclarantes más utilizados es el Xileno, el cual es un agente rápido, requiere alrededor de una hora por cada $5 \mathrm{~mm}$ para completar el proceso sin endurecer en exceso los tejidos, lastimosamente es altamente toxico y controlado (Dey, 2018; Montuenga \& Calvo, 2014). Debido a esto existe en la actualidad un uso creciente de los sustitutos del Xileno en la fase de aclaramiento entre los cuales tenemos a Neo-Celar de la empresa Alemana Merck, Otitix plus de origen italiano, Master clear de Master Tech, entre las más utilizadas que han demostrado encontrarse a la par con el Xileno en las prestaciones de su uso histotecnológico (Moya-Salazar \& Rojas-Zumaran, 2018).

Inclusión, su objetivo principal es el de rellenar los espacios dejados por el agua, es necesario eliminar completamente la sustancia aclarante, para darle a la pieza una estructura homogénea y dureza óptima para realizar buenos cortes (Lester, 2014; Montuenga \& Calvo, 2014; Sy \& Lee-Cyn, 2019).

El medio de impregnación ideal es aquel que sea miscible con el agente aclarante, de consistencia estable y homogénea a los cambios de temperatura, transparente para una adecuada valoración, barato y no toxico durante la manipulación (Dey, 2018).

Existen varias sustancias utilizadas para este proceso. En la actualidad la parafina es la más utilizada, es mezclada con polímeros plásticos que mejoran las características de la misma (Montuenga \& Calvo, 2014). El proceso de inclusión se realiza mediante varios baños en parafina fundida a temperaturas entre 40 y 70 grados centígrados, es importante 
el cambio constante para evitar la disminución de la efectividad y pérdida de las características (Montuenga \& Calvo, 2014; Sadeghipour \& Babaheidarian, 2019).

Se ha establecido un tiempo de entre 3 a 4 horas en parafina para lograr un efecto de impregnación optimo en el tejido en estudio (Dey, 2018).

Técnica de inmunohistoquímica, la aparición, desarrollo y perfeccionamiento de la técnica de inmunohistoquímica, desde sus primeros pasos en la década de los cuarenta del siglo pasado por Coons y colaboradores con el uso de fluorescencia en anticuerpos, la caracterización de la estructura molecular de los anticuerpos por los ganadores del premio nobel Porter y Edelman, hasta el desarrollo y producción de anticuerpos monoclonales por el también ganador del premio nobel en Medicina, Cesar Milstein en 1984, han contribuido como uno de los hitos más notables en la historia de la Medicina. La inmunohistoquímica se ha convertido en la piedra angular junto al estudio morfológico en el diagnóstico, pronóstico y tratamiento de diversas entidades patológicas (Buys \& Hidalgo, 2007; Grillo et al., 2017).

En la actualidad se ha difundido, popularizado y estandarizado en los laboratorios de patología de todo el mundo y su uso se ha vuelto rutinario. Dada su importancia en el diagnóstico el anatomo patólogo está en la obligación de conocer su procedimiento y los múltiples factores que pueden alterar su correcta expresión (Bogen, 2020).

Inmunohistoquímica y el proceso pre analítico, el éxito de un buen resultado en la técnica de inmunohistoquímica, radica en el cuidado de los diferentes pasos en el proceso pre analítico. En esta revisión bibliográfica se analizarán los efectos que provoca en esta técnica el tiempo sin fijación, el tiempo de fijación y el pH del fijador.

Tiempo previo sin fijación, como se había expuesto anteriormente uno de los principales obstáculos para una correcta valoración es el tiempo de isquemia sobre todo de isquemia fría que afecta la preservación del ARNm, acelera la activación de enzimas que degradan tejidos y por consiguiente la perdida en la expresividad de los marcadores inmunohistoquímicos (Bass et al, 2014; Buffart et al., 2021). La literatura aconseja un tiempo máximo de una hora sin fijar para una correcta conservación estructural y expresión de inmuno marcadores (Tresserra et al., 2016; Kanai et al., 2018; Ascierto et al., 2019).

Se ha determinado que los cambios moleculares aparecen desde 15 minutos después de la resección del tejido, la respuesta a la isquemia por la activación de cascadas de fosforilación y desfosforilación de diferentes proteínas desencadenan la perdida de fosfoepítopos necesarios para la reacción antígeno anticuerpo (Neumeister \& Juhl, 2018). No siempre esto es una constante y está determinada por factores como el tipo de tejido de estudio y las condiciones específicas de cada paciente (Khan et al., 2016). Esto 
explicaría la resistencia de ciertos marcadores a los procesos isquémicos (Neumeister \& Juhl, 2018), como son los utilizados en cáncer de mama : KI67 Progesterona, Estrógenos y HER2 que no se ven afectados por la isquemia sin fijación durante la primera hora (Neumeister \& Juhl, 2018).

Tiempo de Fijación, en la mayoría de las guías y protocolos de laboratorio se ha establecido un tiempo de fijación en un rango de 6 a 48 horas (Tresserra et al., 2016; Engel \& Moore, 2011). Se ha demostrado que tiempos cortos de fijación alteran la reacción antígeno anticuerpo debido a que, en la fase de deshidratación del proceso, se genera una fijación alcohólica por un mecanismo de coagulación (Tresserra et al., 2016; Werner et al., 2000). Tiempos prolongados de fijación generan perdida de los puntos antigénicos debido a las características químicas del fijador (Tresserra et al., 2016; Engel $\&$ Moore, 2011). Estos puntos antigénicos disminuyen a los 8 días y se pierden en su totalidad a los 16 días de fijación (Tresserra et al., 2016).

Para muestras grandes son aceptados tiempos de fijación mínimo de 24 a 48 horas con un máximo de 72 horas .En muestras pequeñas el tiempo para lograr una fijación óptima es mínimo de 6 horas pero en ningún caso superar las 72 horas (Dey, 2018; Tresserra et al., 2016).

PH del Fijador, se ha establecido que el medio de fijación influye drásticamente sobre la expresividad de los marcadores de inmunohistoquímica ya que generan enmascaramiento de los puntos antigénicos, este efecto se ve influenciado por el $\mathrm{pH}$ del fijador (Neumeister \& Juhl, 2018; Tresserra et al., 2016).

No existe un consenso sobre un $\mathrm{pH}$ adecuado que modifique las propiedades de los tejidos, algunos autores recomiendan mantener $\mathrm{pH}$ neutro (Bass et al., 2014), otros sostienen que $\mathrm{pH}$ en rangos entre ácido y neutro ( $\mathrm{pH} 5$ a 7) mantienen de mejor manera los puntos antigénicos del tejido en estudio (True, 2014; Engel \& Moore, 2011).

Pollard y Lunny (1987), realizaron un estudio del efecto de la fijación en la expresividad de inmunomarcadores de Linfocitos $\mathrm{T}$, compararon fijadores bufferados con $\mathrm{pH}$ neutro ( $\mathrm{pH} 7,5)$ versus fijadores no bufferados con $\mathrm{pH}$ ácidos ( $\mathrm{pH} 4)$, demostraron que el $\mathrm{pH}$ acido enmascara los puntos antigénicos que se tradujeron en una inmunotinción débil en el tejido.

Por otra parte se ha encontrado beneficioso el uso de $\mathrm{pH}$ alcalinos de los fijadores, logrando una recuperación antigénica en tejidos con fijación excesiva con resultados adecuados para la valoración y sin artefactos de inmunotinción (Marsch et al., 2015). 


\section{Conclusiones}

- La presente revisión demuestra la importancia del proceso pre analítico en sus diferentes fases, y su efecto para obtención de laminillas adecuadas para un correcto análisis. Una adecuada fijación es vital en la fase preanalítica. Muestras sin fijación superiores a 1 hora, muestras con fijación menor a 6 horas o superiores a 72 horas afectan de manera notable la expresividad de marcadores de inmunohistoquímica

- Rangos muy ácidos del fijador generan un efecto negativo con afectación notable en la inmunoexpresividad.

- El Colegio Americano de Patólogos (CAP) establece en sus guías el manejo preanalítico para el procesamiento de muestras de tejido mamario, pero para el resto de los especímenes, no existen protocolos o guías estandarizadas sobre las distintas variables y sus efectos en el proceso pre-analítico, lo que impide una detección oportuna de errores y la corrección de los mismos, generando retrasos en el diagnóstico, pérdida de recursos económicos y material biológico.

- En nuestro país muchos de los procedimientos médicos de manejo, tratamiento y seguimiento de las patologías prevalentes están debidamente regulados por protocolos establecidos y dispuestos por la autoridad sanitaria. Lamentablemente no se cuenta con protocolos propios para el manejo de las muestras histopatológicas por lo que es deseable que las autoridades dispongan la elaboración de normas de manejo del material para estudio anatomo patológico, evitando de esta manera diagnósticos incorrectos, por mal uso de fijadores, tiempos inadecuados o mal transporte de los especímenes

- El conocimiento del proceso pre analítico no solo es una obligación del Anatomo Patólogo como líder del laboratorio sino también del Medico clínico, del cirujano y del personal de apoyo, por lo que se torna importante la socialización de protocolos de manejo de especímenes para lograr un correcto diagnóstico.

\section{Referencias bibliográficas}

Alarcón, M., \& Plaza, L. (2015). Fundamentos Biológicos de Inmunohistoquímica. In Anatomía Patológica y Citológica. (Vol. 5, pp. 17-44). Madrid: Formación Alcala.

Ammerlaan, W., Trouet, J., Sachs, M. C., Guan, P., Carithers, L., Lambert, P., ... Betsou, F. (2018). Small Nucleolar RNA Score: An Assay to Detect Formalin-Overfixed Tissue. Biopreservation and Biobanking, 16(6), 467-476. https://doi.org/10.1089/bio.2018.0042

Anvari, M., \& Gharib, A. (2021). Pre-analytical Practices in the Molecular Diagnostic Tests , A Concise Review. Iranian Journal Of Pathology, 16(1), 1-19. 
Ascierto, P., Bifulco, C., Palmieri, G., Peters, S., Sidiropoulos, N., \& Tumori, I. (2019). Preanalytic Variables and Tissue Stewardship for Reliable Next-Generation Sequencing ( NGS ) Clinical Analysis. The Journal of Molecular Diagnostics, 21(5), 756-767. https://doi.org/10.1016/j.jmoldx.2019.05.004

Atkins, D., Reiffen, K., Tegtmeier, C. L., Winther, H., Bonato, M. S., \& Störkel, S. (2004). Immunohistochemical Detection of EGFR in Paraffin-embedded Tumor Tissues : Variation in Staining Intensity Due to Choice of Fixative and Storage Time of Tissue Sections. The Journal of Histochemistry \& Cytochemistry, 52(7), 893901. https://doi.org/10.1369/jhc.3A6195.2004

Bass, B., Engel, K., Greytak, S., \& Moore, M. (2014). A Review of Preanalytical Factors Affecting Molecular, Protein, and Morphological Analysis of Formalin-Fixed, Paraffin-Embedded (FFPE) Tissue. Archives of Pathology and Laboratory Medicine, 138(11), 1520-1530. https://doi.org/10.5858/arpa.2013-0691-RA

Bellizzi, A. (2020a). An Algorithmic Immunohistochemical Approach to Define Tumor Type and Assign Site of Origin. Adv Anat Pathol., 27(3), 114-163. https://doi.org/10.1097/PAP.0000000000000256.An

Bellizzi, A. (2020b). Immunohistochemistry in the diagnosis and classification of neuroendocrine neoplasms: what can brown do for you? Hum Pathol., 96, 8-33. https://doi.org/10.1016/j.humpath.2019.12.002.Immunohistochemistry

Biobank, G. (2018). Quality Control System in an Obstetrics. Kings College London Journal, 17(1), 27-38. https://doi.org/10.1089/bio.2018.0056

Bloom, K., Branton, P., Crothers, J., Cushman-Vokoun, A., Hicks, D., Nowak, J., ... Vance, G. (2019). Preanalytics and Precision Pathology for Precision Medicine. Archives of Pathology and Laboratory Medicine, 143(11), 1346-1363. https://doi.org/10.5858/arpa.2019-0009-SA

Bogen, S. (2020). A Root Cause Analysis Into The High Error Rate In Clinical Immunohistochemistry. Appl Immunohistochem Mol Morphol., 27(5), 617-636. https://doi.org/10.1097/PAI.0000000000000750.A

Bonin, S., \& Stanta, G. (2020). Pre-analytics and tumor heterogeneity. New Biotechnology, 55(1), 30-35. https://doi.org/10.1016/j.nbt.2019.09.007

Buffart, T., Van den Oord, Van Den Berg, A., Hilhorst, R., Bastiaensen, N., Pruijt, H., ... Verheul, H. (2021). Time dependent effect of cold ischemia on the phosphoproteome and protein kinase activity in fresh - frozen colorectal cancer tissue obtained from patients. Clinical Proteomics, 18(1), 1-12. https://doi.org/10.1186/s12014-02009306-6 
Buys, D., \& Hidalgo, C. (2007). Interpretación básica de inmunohistoquímica. Características generales de diversos anticuerpos y su localización celular y subcelular. Patologia Revista Latinoamericana, 45(3), 126-140.

Cards, F., Acids, N., \& Assays, M. (2018). FTA Cards for Preservation of Nucleic Acids for Molecular Assays. Archives of Pathology and Laboratory Medicine, 142(3), 308-312. https://doi.org/10.5858/arpa.2017-0303-RA

Chung, J., Song, J., Ylaya, K., Sears, J., Choi, L., Cho, H., ... Hewitt, S. (2018). Histomorphological and Molecular Assessments of the Fixation Times Comparing Formalin and Ethanol-Based Fixatives. Journal of Histochemistry \& Cytochemistry, 66(2), 121-135. https://doi.org/10.1369/0022155417741467

Comanescu, M., Annaratone, L., Armento, G. D., Cardos, G., Sapino, A., \& Bussolati, G. (2012). Critical Steps in Tissue Processing in Histopathology. Bentham Science, $6(1), 22-32$.

Dagher, G., Becker, K., Bonin, S., Foy, C., Gelmini, S., Kubista, M., ... Zatloukal, K. (2019). Pre-analytical processes in medical diagnostics: New regulatory requirements and standards. New Biotechnology, 52(1), 121-125. https://doi.org/10.1016/j.nbt.2019.05.002

Dey, P. (2018). Basic and Advanced Laboratory Techniques in Histopathology and Cytology . Singapore: Springer Heidelberg Dordrecht London New York.

Donczo, B., \& Guttman, A. (2018). Biomedical analysis of formalin-fixed, paraffinembedded tissue samples: The Holy Grail for molecular diagnostics. Journal of Pharmaceutical and Biomedical Analysis, 115(1), 125-134. https://doi.org/10.1016/j.jpba.2018.03.065

Donovan, M., \& Cordon, C. (2016). Implementation of a Precision Pathology Program Focused on Oncology-Based Prognostic and Predictive Outcomes. Molecular Diagnosis \& Therapy, 21(2), 115-123. https://doi.org/10.1007/s40291-016-0249-5

Engel, K., \& Moore, H. (2011). Effects of Preanalytical Variables on the Detection of Proteins by Immunohistochemistry in Formalin-Fixed, Paraffin-Embedded Tissue. Archives of Pathology and Laboratory Medicine, 135(5), 1-11.

Fitzgibbons, P. L., Linda, A. B., Lisa, A. F., Alsabeh, R., Regan, S. F., Jeffrey, D. G., ... Swanson, P. E. (2014). Principles of analytic validation of immunohistochemical assays: Guideline from the College of American Pathologists Pathology and Laboratory Quality Center. Archives of Pathology and Laboratory Medicine, 138(11), 1432-1443. https://doi.org/10.5858/arpa.2013-0610-CP 
Gaffney, E., Riegman, P., Grizzle, W., \& Watson, P. (2018). Factors that drive the increasing use of FFPE tissue in basic and translational cancer research Factors that drive the increasing use of FFPE tissue in basic and translational cancer research. Biotechnic \& Histochemistry, https://doi.org/10.1080/10520295.2018.1446101

Grillo, F., Bruzzone, M., Pigozzi, S., Prosapio, S., Migliora, P., Fiocca, R., \& Mastracci, L. (2017). Immunohistochemistry on old archival paraffin blocks : is there an expiry date? J Clin. Pathol., 70(11), 988-993. https://doi.org/10.1136/jclinpath-2017204387

Hammond, M., Hayes, D., Dowsett, M., Allred, D., Hagerty, K., Badve, S., ... Wolff, A. (2010). American Society of Clinical Oncology / College of American Pathologists Guideline Recommendations for Immunohistochemical Testing of Estrogen and Progesterone Receptors in Breast Cancer (Unabridged Version ). Archives of Pathology and Laboratory Medicine, 134(6), 1-14.

Kanai, Y., Nishihara, H., Miyagi, Y., Tsuruyama, T., Taguchi, K., Katoh, H., ... Shibuya, A. (2018). The Japanese Society of Pathology Guidelines on the handling of pathological tissue samples for genomic research: Standard operating procedures based on empirical analyses. Pathology International, 68(1), 63-90. https://doi.org/10.1111/pin.12631

Khan, J., Lieberman, J., \& Lockwood, C. (2016). Variability in , variability out : best practice recommendations to standardize pre-analytical variables in the detection of circulating and tissue microRNAs. Clin Chem Lab Med, 55(5), 608-621. https://doi.org/10.1515/cclm-2016-0471

Lester, S. (2014). Manual of Surgical Pathology. (B. Schmitt, Ed.) (Tercera ed). Philadelphia: Elsevier Inc.

Marsch, A., Truong, J., Mcpherson, M., Junkins-Hopkins, J., \& Elston, D. (2015). A Dermatopathologist's Guide to Troubleshooting Immunohistochemistry - Part 2 : Troubleshooting Immunohistochemical Tests in the Laboratory. AMJ Dermatopathology, 37(9), 665-679.

Mastracci, L., Gambella, A., Bragoni, A., Pigozzi, S., Pastorino, L., Vanni, I., ... Grillo, F. (2019). Coping with formalin banning in pathology: under vacuum long-term tissue storage with no added formalin. Histochemistry and Cell Biology, 151(1), 501-511. https://doi.org/10.1007/s00418-018-1765-7

Mathieson, W., Mommaerts, K., Trouet, J. M., Mathay, C., Guan, P., Carithers, L. J., ... Betsou, F. (2019). Cold Ischemia Score: An mRNA Assay for the Detection of 
Extended Cold Ischemia in Formalin-Fixed , Paraffin- Embedded Tissue. Journal of Histochemistry \& Cytochemistry, 67(3), 159-168. https://doi.org/10.1369/0022155418819967

Maximilian, B. (2021). The Texas Society of Pathologists: molded by the legacy of pathology and focused on excellence in medicine for 100 years and beyond. Baylor University Medical Center Proceedings, 34(1), 199-214. https://doi.org/10.1080/08998280.2020.1812366

Megías, M. \& Molist, P. (2016). Atlas de Histología Vegetal y Animal, Técnicas histológicas. Vigo: Universidad de Vigo.

Metovic, J., Bertero, L., Musuraca, C., Veneziano, F., Annaratone, L., Mariani, S., ... Bussolati, G. (2018). Safe transportation of formalin-fixed liquid-free pathology specimens. Virchows Archiv, 473(1), 105-113.

Miller, R. (2011). Technical Immunohistochemistry: Achieving Reliability and Reproducibility of Immunostains. Society for Applied Immunohistochemistry, 1(1), $1-56$.

Montuenga, L. \& Calvo, A. (2014). Téctinicas en Histología y Biologia Celular (Segunda Ed). Barcelona: Elsevier Inc.

Moorchung, N., \& Consultant, S. (2019). Rapid ' technological lock in ' and pathology Is technology moving too fast? Malaysian J Pathol, 41(1), 1-5.

Moya-Salazar, J., \& Rojas-Zumaran, V. (2018). Análisis de la problemática del xileno en los laboratorios Sudamericanos de citología. Revista Latinoamericana de Patología Clínica y Medicina de Laboratorio, 65(3), 150-158.

Neumeister, V., \& Juhl, H. (2018). Tumor Pre-Analytics in Molecular Pathology: Impact on Protein Expression and Analysis. Current Pathobiology Reports, 1(6), 265-274.

Parra-Medina, R., \& Polo, J. (2017). Inmunofluorescencia en tejidos fijados y preservados en parafina (IF-P). Una mirada desde la patología quirúrgica. Repertorio de Medicina y Cirugía, 26(4), 202-207. https://doi.org/10.1016/j.reper.2017.08.009

Pollard, K., \& Lunny, D. (1987). Fixation , Processing , and Immunochemical on Preservation Antigens in Paraffin-embedded Tissue Reagent Membrane Effects. The Journal of Histochemistry and Citochemistry, 35(11), 1329-1338.

Rourke, M., \& Padula, M. (2016). Analysis of formalin-fixed, paraffin-embedded (FFPE) tissue via proteomic techniques and misconceptions of antigen retrieval. Bio Techniques, 1998(3), 229-238. https://doi.org/10.2144/000114414 
Sadeghipour, A., \& Babaheidarian, P. (2019). Making Formalin-Fixed, Paraffin Embedded Blocks. In Biobanking: Methods and Protocols, Methods in Molecular Biology, 1897, 253-268.

Schmeller, J., Wessolly, M., Mairinger, E., Borchert, S., Hager, T., Mairinger, T., ... Mairinger, F. (2019). Setting out the frame conditions for feasible use of FFPE derived RNA. Pathology - Research and Practice, 215(2), 381-386. https://doi.org/10.1016/j.prp.2018.12.027

Shivani, R., \& Lin, F. (2017). Application of Immunohistochemistry in. Archives of Pathology and Laboratory Medicine, 141(8), 1014-1032. https://doi.org/10.5858/arpa.2016-0518-RA

Socias, E., Med, J., Socias, M., Artiga, M., Bahamonde, O., Belar, O., ... Campo, V. (2019). In search of an evidence - based strategy for quality assessment of human tissue samples : report of the tissue Biospecimen Research Working Group of the Spanish Biobank Network. Journal of Translational Medicine, 17(1), 1-14. https://doi.org/10.1186/s12967-019-2124-8

Stradleigh, T., \& Ishida, A. (2015). Progress in Retinal and Eye Research Fixation strategies for retinal immunohistochemistry. Prog Retin Eye Res, 48, 1-22.

Sy, J., \& Lee-Cyn, A. (2019). Microtomy: Cutting Formalin-Fixed, Paraffin-Embedded Sections. In Biobanking: Methods and Protocols, Methods in Molecular Biology, 897, 269-278.

Ter, M., Bojan, Š., \& Verani, P. (2017). How to obtain good morphology and antigen detection in the same tissue section? Protoplasma, 254(5), 1931-1939. https://doi.org/10.1007/s00709-017-1085-0

Teruya-Feldstein, J. (2011). The Immunohistochemistry Laboratory. Archives of Pathology and Laboratory Medicine, 134(2), 1659-1665. https://doi.org/10.1043/2009-0582-RAR1.1

Thway, K., Wren, D., Lee, J., Thompson, L., Fisher, C., \& Gonzalez, D. (2017). Evaluation of the optimal provision of formalin- fi xed, paraf fi n-embedded material for reverse transcription-PCR in soft-tissue tumour diagnosis. J Clin. Pathol, 70(1), 20-24. https://doi.org/10.1136/jclinpath-2016-203691

Tresserra, F., Angeles, M., Lanao, M., \& Soler, M. T. (2016). Manejo de las muestras para test inmunohistoquímicos, moleculares y genéticos en el cáncer de mama. Revista de Senología y Patología Mamaria, 29(1), 26-31.

True, L. (2014). Methodological requirements for valid tissue-based biomarker studies 
that can be used in clinical practice. Virchows Arch, 464(3), 257-263. https://doi.org/10.1007/s00428-013-1531-0

Uguen, A., \& Guibourg, B. (2016). Standardized fixation process is crucial to permit molecular analyses in formalin-fixed and paraffin-embedded melanoma samples. Clin Chem Lab Med, 54(11), 2. https://doi.org/10.1515/cclm-2016-0397

Vaquero, M. (2012). Manual de Calidad de Inmunohistoquímica en Anatomía Patológica. (H. U. Donostia, Ed.). San Sebastian, España: Hospital Universitario Donostia.

Werner, M., Chott, A., Fabiano, A., \& Battifora, H. (2000). Effect of Formalin Tissue Fixation and Processing on Immunohistochemistry, Am J Surg Pathol, 24(7), 10161019.

\section{ULiencia}


El artículo que se publica es de exclusiva responsabilidad de los autores y no necesariamente reflejan el pensamiento de la Revista Anatomía Digital.

\section{Ligital}

El artículo queda en propiedad de la revista y, por tanto, su publicación parcial y/o total en otro medio tiene que ser autorizado por el director de la Revista Anatomía Digital.
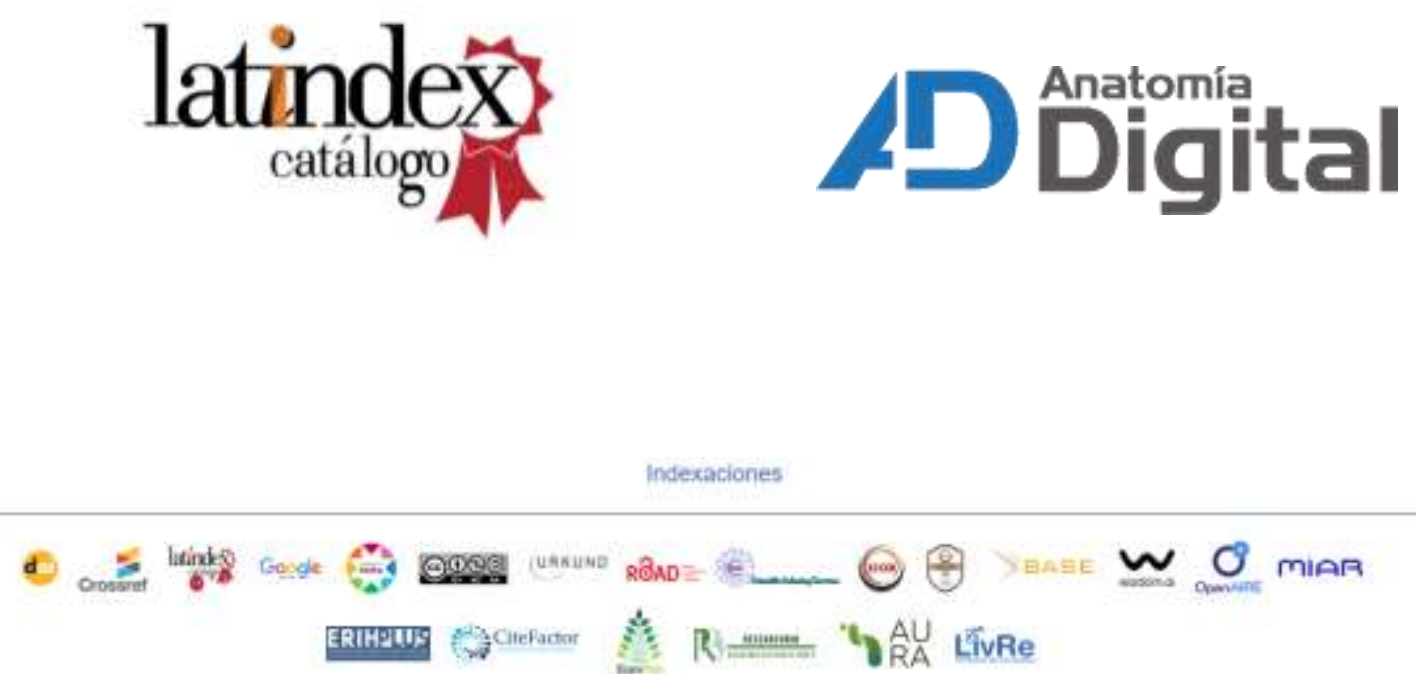